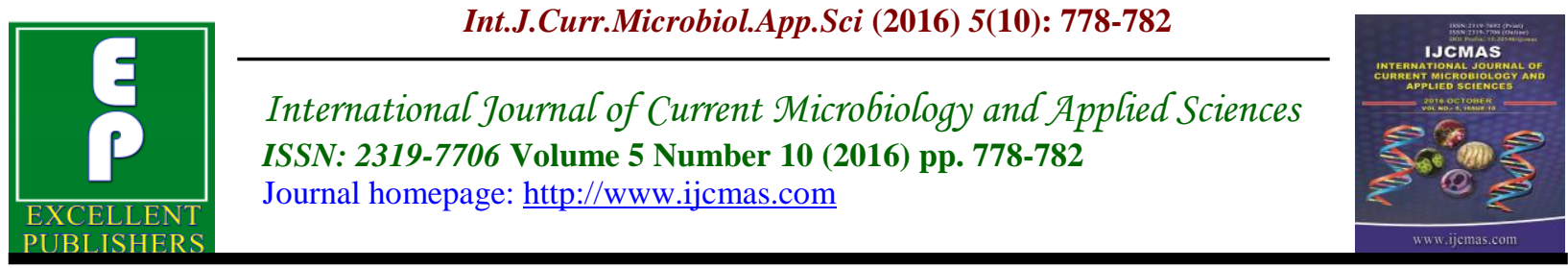

Original Research Article

http://dx.doi.org/10.20546/ijcmas.2016.510.084

\title{
Hepatitis B Surface Antigen Detection by Enhanced Chemiluminiscence A Pilot Study to Determine the Gray Zone Results
}

\author{
Ryhan Bashir $^{1 *}$, M.H. Sharif ${ }^{2}$, Vidhya $^{1}$ and Varsha Saxena ${ }^{1}$ \\ Department of Microbiology, YMCH, Mangalore, India \\ Department of Pathology, YMCH, Mangalore, India \\ *Corresponding author
}

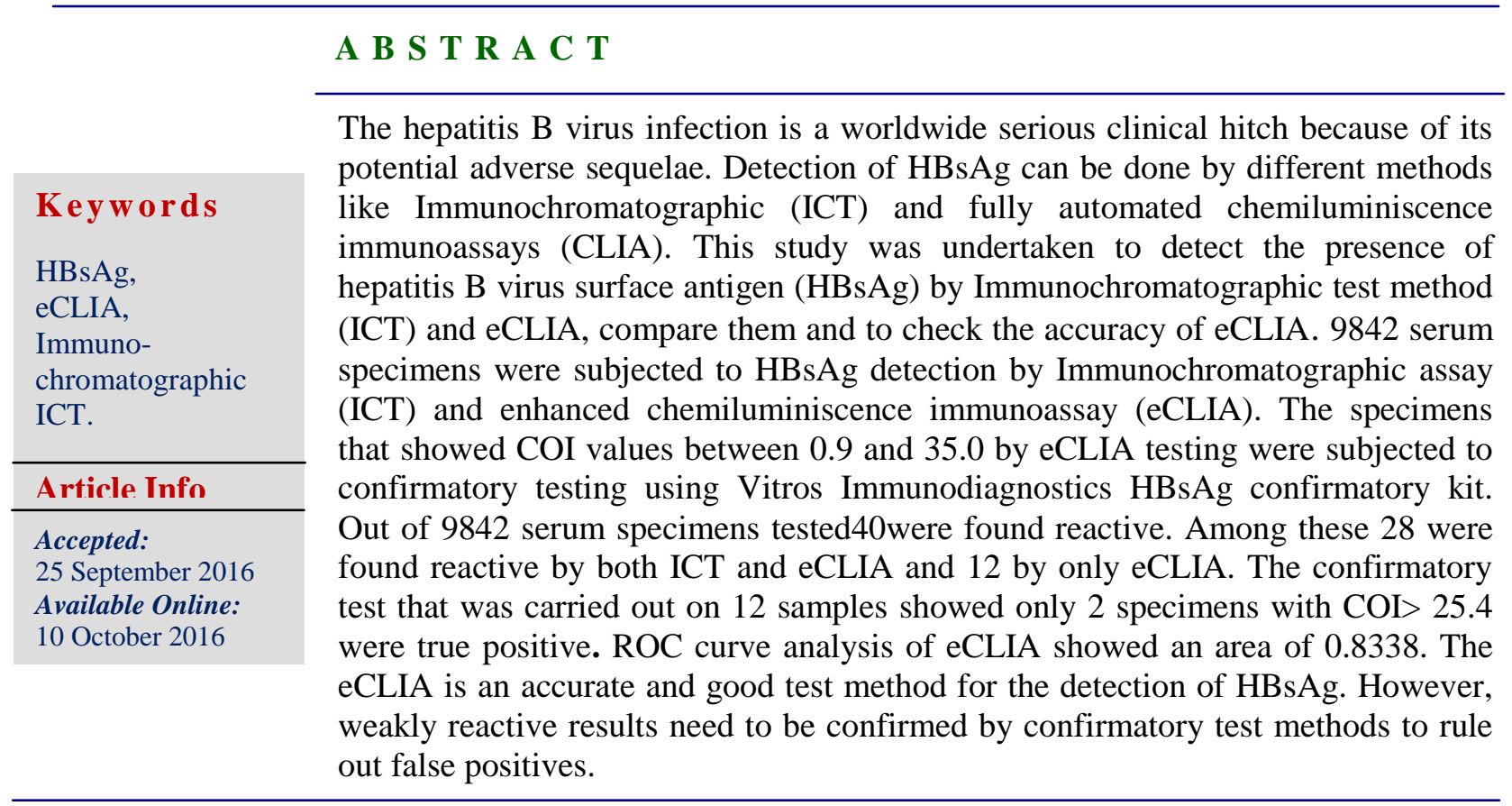

\section{Introduction}

The hepatitis $\mathrm{B}$ virus infection is a worldwide serious clinical hitch because of its potential adverse sequelae. Approximately two billion people have been infected worldwide and over 5, 20,000 die each year (Mohammad-Hassan et al., 2014). Hepatitis B virus (HBV) is a small DNA virus that belongs to the Hepadnaviridae family. Hepatitis B virus (HBV) infection leads to a wide spectrum of liver diseases ranging from acute to chronic hepatitis, cirrhosis, and hepatocellular carcinoma (HCC). Most adults infected with the virus convalesce, but 5-10\% are unable to clear the virus and become chronically infected (Gupta et al., 2012). Hepatitis B surface antigen (HBsAg) has been the principal target for laboratory testing to identify active infection by HBV.

The accurate detection of HBsAg is crucial for early diagnosis and treatment of $\mathrm{HBV}$ 
infection. The balance between virus biology and a host's immune system along with the indirect expression of transcriptionally active covalently closed circular DNA (cccDNA) decide the serum levels of HBsAg and not the viral replication (Hua et al., 2012; Mauro et al., 2012). Quantification of HBsAg can be done by different methods like radio-immunoassays (RIA) and fully automated chemiluminiscence immunoassays (CLIA). The CLIA is more sensitive, specific, and can detect all circulating forms of HBsAg as well as mutants (Gupta et al., 2015).

This study was undertaken to detect the presence of hepatitis B virus surface antigen (HBsAg) by Immunochromatographic test method (ICT) and eCLIA, compare them and to confirm the weakly reactive results.

\section{Materials and Methods}

The study was done in the microbiology department of a tertiary care teaching hospital. The serum specimens received over a period of 10 months were subjected to HBsAg detection by Immunochromatographic assay (ICT) and enhanced chemiluminiscence immunoassay [eCLIA (Vitros ECiQ, J\&J)]. The specimens that showed COI values between 0.9 and 35.0 by eCLIA testing were subjected to confirmatory testing to evaluate the threshold for $\mathrm{HBsAg}$ confirmation using Vitros Immunodiagnostics HBsAg confirmatory kit.

\section{ICT method}

Rapid screening method (SD BIOLINE HBsAg) is an in-vitro Immunochromatographic, one step assay designed for qualitative determination of $\mathrm{HBsAg}$ in human serum or plasma. This test cassette contains a membrane strip, which is precoated.
Enhanced chemiluminiscence immunoassay (eCLIA)

This test method is used in vitro qualitative determination of hepatitis B surface antigen (HBsAg) in human serum and plasma. The serum samples were processed in the VitrosEci system as per manufacturer's instructions. The serum samples which showed a Cut off index of 0.9 to 35.0 were tested to rule out false positive results, by using the HBsAg confirmatory kit.

\section{Results and Discussion}

A total of 9842 serum specimens were tested, of which 40 were found reactive. Among these 28 were found reactive by both ICT and eCLIA and 12 by only eCLIA(Table1). The confirmatory test that was carried out on specimens with COI values between 0.9 and 35.0, showed only 2 specimens with COI $>25.4$ were true positive. The analysis of the data of samples reactive by eCLIA alone and confirmatory test method was done by plotting a receiver operating characteristic curve (ROC curve) shown in (Figure 1).

HBV surface antigen (HBsAg) is the recognized serological marker used routinely for the diagnosis of acute or chronic HBV infection, the screening of blood or organ donors, and the surveillance of persons at risk of acquiring or transmitting HBV.

HBsAg is the first marker to appear during acute $\mathrm{HBV}$ infection. The HBsAg positivity indicates the presence of $\mathrm{HBV}$ infection, which eventually stimulates the production of anti-HBV antibodies. Once the anti-HBV antibodies emerge, HBsAg disappears. However, there is a period before the appearance of anti-HBV antibodies during which neither HBsAg nor anti-HBV antibodies can be detected in the patient's 
blood, making it difficult to detect infection. However, high-sensitivity assays have made it is likely to detect trace amounts of HBsAg, allowing HBV infection to be diagnosed earlier in the evolution of the disease, which may lead to more effective treatment. ${ }^{3} \mathrm{HBsAg}$ detection is done by most of the laboratories by ICT method, which may fail to detect it when the antigenemia is low in serum, resulting in under diagnosis (Safia et al., 2014). Thus the surfacing of new methods for detecting trace levels of HBsAg represents a significant enhancement in the diagnosis of HBV. However, such improvements in sensitivity often are associated with more false-positive results. Hence it is required to check the validity of initial reactive samples, either by repeating by the same test, and/or followed up with confirmatory testing. It has been reported that differences exist in the sensitivity of various instruments and reagents for detecting serum HBV markers, more so with samples showing weakly reactive results, especially in initial testing (chen et al., 2006). It is particularly important in geographic locations, where the prevalence is high.

Table.1 Comparison of results of ICT and eCLIA

\begin{tabular}{|l|l|l|}
\hline Result of HBsAg detection & ICT & eCLIA \\
\hline $\begin{array}{l}\text { Reactive } \\
\text { COI }>35.0\end{array}$ & 28 & 28 \\
\hline $\begin{array}{l}\text { Weakly reactive } \\
\text { COI }-1.0-35.0\end{array}$ & $\mathbf{0 0}$ & $\mathbf{0 4}$ \\
\hline $\begin{array}{l}\text { Borderline } \\
\text { COI-0.9-1.0 }\end{array}$ & $\mathbf{0 0}$ & $\mathbf{0 8}$ \\
\hline $\begin{array}{l}\text { Negative } \\
\text { COI }<0.9\end{array}$ & $\mathbf{9 8 1 4}$ & $\mathbf{9 8 0 2}$ \\
\hline
\end{tabular}

Fig.1 ROC curve for eCLIA and Confirmatory test

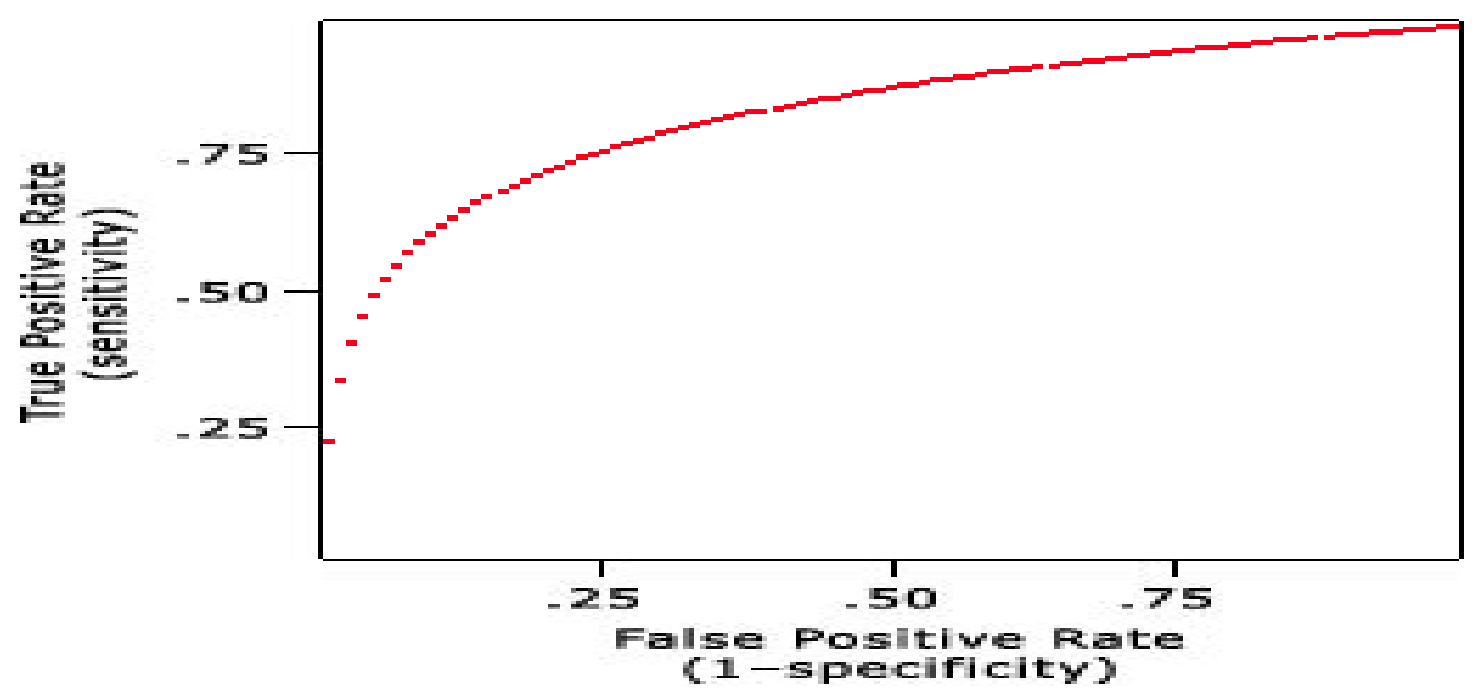

ROC Curve for $\mathrm{y}=0.17 \operatorname{Ln}(\mathrm{x})+1$

Area under curve $=0.8338$ 
In India, the estimated rate of chronic HBV infection is 40 million. Studies have suggested that a confirmatory testing needs to be done for weakly reactive results by chemiluminiscence as the sensitivity of this technique is very high, hence chances of false positives also becomes high. The assay is also specific that strongly reactive results need not be tested for confirmation; thereby avoiding the cost (Robert, 2006). Similar recommendations have been made for weakly reactive results of $\mathrm{HCV}$ by the Centers for Disease Control. Weakly positive results are also more likely to be falsely positive with other infectious disease assays, including anti-HIV and anti-HBc (Miriam et al., 2003).

Tested a total of 9842 serum specimens of which $40(0.45 \%)$ were found reactive. The Immuno chromatographic test could not detect 12 samples that were reactive by eCLIA. This may be probably due to the lesser sensitivity of the assay or low level antigenemia in the samples. These 12 specimens showed a COI of <35.0andwere repeat tested with the confirmatory kit, to rule out false positives and only two turned out to be true positive. The sensitivity of eCLIA is very high, so there is a probability of false positives. As per manufacturer a $\mathrm{COI}$ of $>=1.0$ is taken as reactive. However, several studies have reported different $\mathrm{CO}$ indices which may be considered as true positive. The gray zone, where the reactivity is due to some other factors present in the serum, is different in different populations. We found that, the samples that showed a COI of $>25.0$ were true positives and probably the gray zone varied from COI of 0.9 to 25.0 .

A study from China has reported that weakly reactive results are more common in HBsAg negative group than those in positive group and found a gay zone of COI from
$<2.0$ up to 12.1 (Hongxia et al., 2012). Another study using a different system for sample analysis (Siemens Healthcare Diagnostics, Tarrytown, NY) suggested that confirmatory testing is essential for samples that show weakly reactive results in initial testing. ${ }^{11}$ We analyzed our data to find the usefulness of eCLIA testing of serum samples to detect HBsAg against the standard confirmatory test method by a Receiver Operating Characteristic (ROC) curve. It was found that the area under the curve is 0.8338 , which depicts that eCLIA is an accurate and good test method for the determination of HBsAg in serum samples.

In conclusion, HBsAg testing is very commonly required in acute or chronic HBV disease to diagnose and to predict the prognosis of condition. We conclude that, eCLIA is an accurate and good test method for the detection of HBsAg in these patients. However, weakly reactive results need to be confirmed by confirmatory test methods to rule out false positives. Larger population based studies may be needed to determine the exact gray zone for interpretation by eCLIA accurate and good test method for the determination of HBsAg in serum samples.

\section{References}

Chen, Y., Wu, W., Li, L.j., et al. 2006. Comprision of the results for threeautomaated immunoassay system in determining serum HBV marker. Clin. Chim. Acta, 372: 129 -133.

Dan, C., Lawrence, K., Qiang, L. 2005. Evaluation of two chemiluminescent immunoassays of ADVIA centaur for hepatitis B serology markers. Clin. Chim. Acta, 355: 41-45.

Gupta, E., A. Kumar, A. Choudhary, M. Kumar, S.K. Sarin. 2012. Serum hepatitis B surface antigen levels 
correlate with high serum HBV DNA levels in patients with chronic hepatitis B: A cross-sectional study. Indian J. Med. Microbiol., 30(2): 150-4.

Gupta, E., P. Pandey, A. Kumar, M.K. Sharma, S.K. Sarin. 2015. Correlation between two chemiluminescence based assays for quantification of hepatitis B surface antigen in patients with chronic hepatitis B infection. Indian J. Med. Microbiol., 33(1): 96100.

Hongxia, N., Dongdong, L., Xiahui, B., et al. 2012.Compare HBsAg cutoff index values of borderline or initially weakly reactive samples with neutralization results or electrochemiluminescence immunoassays in china. African $J$. Microbiol. Res., 6(12): 3024-28.

Hua, S., Yan, L., Wan, Zhou. X., Xin, Z. 2012. Increased need for testing to confirm initial weakly reactive results for hepatitis B virus surface antigen. Lab. Med., 43(4): 15-17.

Mauro, V., Pietro, L. 2012. Clinical Implications of HBsAg Quantification in Patients with Chronic Hepatitis B. Saudi J. Gastroenterol., 18: 81-6.

Miriam, J., Wendi, L., Lyn, F. 2003. Guidelines for laboratory testing and result reporting of antibody to hepatitis $\mathrm{C}$ virus. Morbidity and mortality weekly report (CDC), 52(3): 1-13.

Mohammad-Hassan, K.A., Mir-Davood, O., Yousef, R., Arsalan, G. 2014. Diagnostic validity of the chemiluminescent method compared to polymerase chain reaction for hepatitis B virus detection in the routine clinical diagnostic laboratory. Adv. Biomed. Res., 3: 116-119.

Robert, D.D. 2006. Hepatitis B Surface Antigen (HBsAg) Assays-Are They Enough for Their Current Uses? Clin. Chem., 52(8): 1457-1459.

Safia, B., Waquaruddin, A., Syed Ejaz, A. 2014. Comparison of Rapid Test with ELISA for the Detection of Hepatitis B Surface Antibodies. Pak. J. Med. Res., 53(3): 60-62.

\section{How to cite this article:}

Ryhan Bashir, M.H. Sharif, Vidhya and Varsha Saxena. 2016. Hepatitis B Surface Antigen Detection by Enhanced Chemiluminiscence-A Pilot Study to Determine the Gray Zone Results. Int.J.Curr.Microbiol.App.Sci. 5(10): 778-782. doi: http://dx.doi.org/10.20546/ijcmas.2016.510.084 